\title{
Fractional Solutions of Bessel Equation with $N$-Method
}

\author{
Erdal Bas, Resat Yilmazer, and Etibar Panakhov \\ Department of Mathematics, Firat University, 23119 Elazig, Turkey \\ Correspondence should be addressed to Erdal Bas; erdalmat@yahoo.com
}

Received 30 April 2013; Accepted 16 July 2013

Academic Editors: R. M. Guedes, Z. Mukandavire, and R. Wangkeeree

Copyright (C) 2013 Erdal Bas et al. This is an open access article distributed under the Creative Commons Attribution License, which permits unrestricted use, distribution, and reproduction in any medium, provided the original work is properly cited.

This paper deals with the design fractional solution of Bessel equation. We obtain explicit solutions of the equation with the help of fractional calculus techniques. Using the $N$-fractional calculus operator $N^{v}$ method, we derive the fractional solutions of the equation.

\section{Introduction, Definitions, and Preliminaries}

Fractional calculus has an important place in the field of math. Firstly, L'Hospital and Leibniz were interested in the topic in 1695, [1]. Fractional calculus is an area of applied mathematics that deals with derivatives and integrals of arbitrary orders and their applications in science, engineering, mathematics, economics, and other fields. The seeds of fractional derivatives were planted over 300 years ago. Since then many efficient mathematicians of their times, such as N. H. Abel, M. Caputo, L. Euler, J. Fourier, A. K. Grunwald, J. Hadamard, G. H. Hardy, O. Heaviside, H. J. Holmgren, P. S. Laplace, G. W. Leibniz, A. V. Letnikov, J. Liouville, and B. Riemann, have contributed to this field; all these references can be seen in [1-5]. The mathematics involved appeared very different applications of this field. Fractional calculus has been applied to almost every field of science. They are viscoelasticity, electrical engineering, electrochemistry, biology, biophysics and bioengineering, signal and image processing, mechanics, mechatronics, physics, and control theory. During the last decade, Samko et al. [4], Nishimoto [6-12], and Podlubny [3] have been helpful in introducing the field to engineering, science, economics and finance, and pure and applied field. Furthermore, there were many studies in this field [5, 13-16]. Various scientists have studied that concept. The progress in this field continues $[2-4,6-12,17-$ 20].

$\mathrm{N}$-Fractional calculus is a very interesting method because this method is applied to singular equation. Note that fractional solutions can be obtained for kinds of singular equation via this method $[6-12,17]$. In this paper, our aim is to apply the same way for singular Sturm-Liouville equation with Bessel potential and find fractional solutions of this equation. Furthermore, we give some applications and their graphs of fractional solutions of the equation.

Now, consider the following the Bessel equation:

$$
x \frac{d^{2} z}{d x^{2}}+\frac{d z}{d x}+\left[\lambda x-\frac{p^{2}}{x}\right] z=0, \quad 0<x \leq 1,
$$

where $\lambda$ and $p$ are real numbers. By means of the substitution $y=\sqrt{x z}$ (1) reduces to the form

$$
\frac{d^{2} y}{d x^{2}}+\left(\lambda-\frac{p^{2}-1 / 4}{x^{2}}\right) y=0 .
$$

Bessel equation for having the analogous singularity is given in [21].

The differintegration operators and their generalizations $[6-11,17,18]$ have been used to solve some classes of differential equations and fractional differential equations.

Two of the most commonly encountered tools in the theory and applications of fractional calculus are provided by the Riemann-Liouville operator $R_{z}^{v}(v \in \mathbb{C})$ and the Weyl operator $W_{z}^{v}(v \in \mathbb{C})$, which are defined by $[17,19]$. 
Consider

$$
\begin{aligned}
& R_{z}^{v} f(z) \\
& = \begin{cases}\frac{1}{\Gamma(v)} \int_{0}^{z}(z-t)^{v-1} f(t) d t & (\operatorname{Re}(v)>0), \\
\frac{d^{n}}{d z^{n}} R_{z}^{v+n} f(z) & (-n<\operatorname{Re}(v) \leq 0 ; n \in \mathbb{N}),\end{cases}
\end{aligned}
$$

$W_{z}^{v} f(z)$

$$
= \begin{cases}\frac{1}{\Gamma(v)} \int_{z}^{\infty}(t-z)^{v-1} f(t) d t & (\operatorname{Re}(v)>0), \\ \frac{d^{n}}{d z^{n}} W_{z}^{v+n} f(z) & (-n<\operatorname{Re}(v) \leq 0 ; n \in \mathbb{N})\end{cases}
$$

provided that the defining integrals in (3) and (4) exist, $\mathbb{N}$ being the set of positive integers.

Definition 1 (cf. [6-10, 12, 20]). Let

$$
D=\left\{D^{-}, D^{+}\right\}, \quad C=\left\{C^{-}, C^{+}\right\},
$$

where $C^{-}$is a curve along the cut joining two points $z$ and $-\infty+i \operatorname{Im}(z), C^{+}$is a curve along the cut joining two points $z$ and $\infty+i \operatorname{Im}(z), D^{-}$is a domain surrounded by $C^{-}$, and $D^{+}$ is a domain surrounded by $C^{+}$. (Here $D$ contains the points over the curve C.) D),

Moreover, let $f=f(z)$ be a regular function in $D(z \in$

$$
\begin{aligned}
f_{\nu}(z)= & (f(z))_{\nu}=\frac{\Gamma(\nu+1)}{2 \pi i} \int_{C} \frac{f(t) d t}{(t-z)^{\nu+1}} \\
& \left(\nu \in \mathbb{R} \backslash \mathbb{Z}^{-} ; \mathbb{Z}^{-}=\{\ldots,-3,-2,-1\}\right), \\
f_{-n}(z)= & \lim _{\nu \rightarrow-n} f_{\nu}(z) \quad\left(n \in \mathbb{Z}^{+}\right),
\end{aligned}
$$

where $t \neq z$,

$$
\begin{array}{ll}
-\pi \leq \arg (t-z) \leq \pi & \text { for } C^{-}, \\
0 \leq \arg (t-z) \leq 2 \pi & \text { for } C^{+} .
\end{array}
$$

Then $f_{\nu}(z)(\nu>0)$ is said to be the fractional derivative of $f(z)$ of order $v$ and $f_{v}(z)(\nu<0)$ is said to be the fractional integral of $f(z)$ of order $-v$, provided (in each case) that $\left|f_{\nu}(z)\right|<\infty(\nu \in \mathbb{R})$.

Finally, let the fractional calculus operator (Nishimoto's operator) $N^{v}$ be defined by (cf. [6-10])

$$
N^{\nu}=\left(\frac{\Gamma(\nu+1)}{2 \pi i} \int_{C} \frac{d t}{(t-z)^{\nu+1}}\right) \quad\left(\nu \notin \mathbb{Z}^{-}\right)
$$

with

$$
N^{-n}=\lim _{\nu \rightarrow-n} N^{\nu} \quad\left(n \in \mathbb{Z}^{+}\right) .
$$

We find it to be worthwhile to recall here the following useful lemmas and properties associated with the fractional differintegration which was defined earlier (cf. e.g., [6-10, 12]).
Lemma 2 (linearity property). If the functions $f(z)$ and $g(z)$ are single-valued and analytic in some domain $\Omega \subseteq \mathbb{C}$, then

$$
\begin{array}{r}
\left(h_{1} f(z)+h_{2} g(z)\right)_{v}=h_{1} f_{v}(z)+h_{2} g_{v}(z) \\
(v \in \mathbb{R} ; z \in \Omega)
\end{array}
$$

for any constants $h_{1}$ and $h_{2}$.

Lemma 3 (index law). If the function $f(z)$ is single-valued and analytic in some domain $\Omega \subseteq \mathbb{C}$, then

$$
\begin{aligned}
& \left(f_{\sigma}(z)\right)_{\nu}=f_{\sigma+v}(z)=\left(f_{\nu}(z)\right)_{\sigma} \\
& \left(f_{\sigma}(z) \neq 0 ; f_{\nu}(z) \neq 0 ; \sigma, v \in \mathbb{R} ; z \in \Omega\right) .
\end{aligned}
$$

Lemma 4 (generalized Leibniz rule). If the functions $f(z)$ and $g(z)$ are single-valued and analytic in some domain $\Omega \subseteq$ $\mathbb{C}$, then

$$
\begin{array}{r}
(f(z) \cdot g(z))_{\nu}=\sum_{n=0}^{\infty}\left(\begin{array}{l}
\nu \\
n
\end{array}\right) f_{\nu-n}(z) \cdot g_{n}(z) \\
(\nu \in \mathbb{R} ; z \in \Omega),
\end{array}
$$

where $g_{n}(z)$ is the ordinary derivative of $g(z)$ of order $n$ ( $n \in$ $\mathbb{N}_{0}:=\mathbb{N} \cup\{0\}$ ), being tacitly assumed (for simplicity) that $g(z)$ is the polynomial part (if any) of the product $f(z) g(z)$.

Property 1. For a constant $\lambda$,

$$
\left(e^{\lambda z}\right)_{\nu}=\lambda^{\nu} e^{\lambda z} \quad(\lambda \neq 0 ; \nu \in \mathbb{R} ; z \in \mathbb{C}) .
$$

Property 2. For a constant $\lambda$,

$$
\left(e^{-\lambda z}\right)_{\nu}=e^{-i \pi \nu} \lambda^{\nu} e^{-\lambda z} \quad(\lambda \neq 0 ; \nu \in \mathbb{R} ; z \in \mathbb{C}) .
$$

Property 3. For a constant $\lambda$,

$$
\begin{aligned}
& \left(z^{\lambda}\right)_{\nu}=e^{-i \pi \nu} \frac{\Gamma(\nu-\lambda)}{\Gamma(-\lambda)} z^{\lambda-\nu} \\
& \left(\nu \in \mathbb{R} ; z \in \mathbb{C} ;\left|\frac{\Gamma(\nu-\lambda)}{\Gamma(-\lambda)}\right|<\infty\right) .
\end{aligned}
$$

Now, let apply $N$-fractional method to nonhomogeneous Bessel equation.

\section{The $N^{\nu}$-Method Applied to Bessel Equation}

Theorem 5. Let $y \in\left\{y: 0 \neq\left|y_{\nu}\right|<\infty ; \nu \in \mathbb{R}\right\}$ and $f \in\{f$ : $\left.0 \neq\left|f_{\nu}\right|<\infty ; \nu \in \mathbb{R}\right\}$. We consider the nonhomogeneous Bessel equation:

$$
L[y, x, \lambda, p]=y_{2}+y\left[\lambda-\frac{p^{2}-1 / 4}{x^{2}}\right]=f \quad(0<x \leq 1),
$$


and it has particular solutions of the forms

$$
\begin{aligned}
& y^{l}=x^{p+1 / 2} e^{-i \sqrt{\lambda} x} \\
& \times\left\{\left[\left(f x^{1 / 2-p} e^{i \sqrt{\lambda} x}\right)_{-p-1 / 2} e^{-2 i \sqrt{\lambda} x} x^{p-1 / 2}\right]_{-1}\right. \\
& \left.\times e^{2 i \sqrt{\lambda} x} x^{-p-1 / 2}\right\}_{p-1 / 2}, \\
& y^{\imath l}=x^{p+1 / 2} e^{i \sqrt{\lambda} x} \\
& \times\left\{\left[\left(f x^{1 / 2-p} e^{-i \sqrt{\lambda} x}\right)_{-p-1 / 2} e^{2 i \sqrt{\lambda} x} x^{p-1 / 2}\right]_{-1}\right. \\
& \left.\times e^{-2 i \sqrt{\lambda} x} x^{-p-1 / 2}\right\}_{p-1 / 2}, \\
& y^{l n l}=x^{-p+1 / 2} e^{-i \sqrt{\lambda} x} \\
& \times\left\{\left[\left(f x^{1 / 2+p} e^{i \sqrt{\lambda} x}\right)_{p-1 / 2} e^{-2 i \sqrt{\lambda} x} x^{-p-1 / 2}\right]_{-1}\right. \\
& \left.\times e^{2 i \sqrt{\lambda} x} x^{p-1 / 2}\right\}_{-p-1 / 2}, \\
& y^{i v}=x^{-p+1 / 2} e^{i \sqrt{\lambda} x} \\
& \times\left\{\left[\left(f x^{1 / 2+p} e^{-i \sqrt{\lambda} x}\right)_{p-1 / 2} e^{2 i \sqrt{\lambda} x} x^{-p-1 / 2}\right]_{-1}\right. \\
& \left.\times e^{-2 i \sqrt{\lambda} x} x^{p-1 / 2}\right\}_{-p-1 / 2},
\end{aligned}
$$

where $y_{2}=d^{2} y / d x^{2}, y=y(z)(z \in \mathbb{C}), f=f(z)$ (an arbitrary given function), and $p, \lambda$ are given constants.

Remark 6. The cases $p=0$ of (19) and (20) coincide with those (17) and (18).

Proof. Set

$$
y=x^{\eta} \psi, \quad \psi=\psi(x)
$$

Thus

$$
\begin{gathered}
y_{1}=\eta x^{\eta-1} \psi+x^{\eta} \psi_{1} \\
y_{2}=\eta(\eta-1) x^{\eta-2} \psi+2 \eta x^{\eta-1} \psi_{1}+x^{\eta} \psi_{2} .
\end{gathered}
$$

Putting (21) and (22) in (16), we obtained

$$
\begin{aligned}
& \psi_{2} x^{\eta}+\psi_{1} 2 \eta x^{\eta-1}+\psi \eta(\eta-1) x^{\eta-2} \\
& +x^{\eta} \psi\left[\lambda-\frac{p^{2}-1 / 4}{x^{2}}\right]=f .
\end{aligned}
$$

With some rearrangement of the terms in (23), we have

$$
\psi_{2} x+\psi_{1} 2 \eta+\psi\left[x^{-1}\left(\eta^{2}-\eta+\frac{1}{4}-p^{2}\right)+\lambda x\right]=f x^{1-\eta} .
$$

Here, we choose $\eta$ such that

$$
\eta^{2}-\eta+\frac{1}{4}-p^{2}=0
$$

That is,

$$
\eta=\frac{1}{2} \pm p
$$

(I) Let $\eta=p+(1 / 2)$. From (21) and (24), we have

$$
\begin{gathered}
y=x^{p+(1 / 2)} \psi \\
\psi_{2} x+\psi_{1}(2 p+1)+\psi \lambda x=f x^{(1 / 2)-p} .
\end{gathered}
$$

Set

$$
\psi=e^{\mu x} \phi, \quad \phi=\phi(x) .
$$

Rewrite (28) in the form

$$
\left(e^{\mu x} \phi\right)_{2} x+\left(e^{\mu x} \phi\right)_{1}(2 p+1)+e^{\mu x} \phi \lambda x=f x^{(1 / 2)-p} .
$$

At this point, differentiating $e^{\mu x} \phi$ two times,

$$
\begin{gathered}
\left(e^{\mu x} \phi\right)_{1}=e^{\mu x}\left(\mu \phi+\phi_{1}\right), \\
\left(e^{\mu x} \phi\right)_{2}=e^{\mu x}\left(\mu^{2} \phi+2 \mu \phi_{1}+\phi_{2}\right),
\end{gathered}
$$

and substituting from (29) and (31) in (30), we can express (30) as

$$
\begin{aligned}
& \phi_{2} x+\phi_{1}(2 \mu x+2 p+1) \\
& \quad+\phi\left(2 p \mu+\mu+x\left(\mu^{2}+\lambda\right)\right)=f x^{(1 / 2)-p} e^{-\mu x} .
\end{aligned}
$$

Choose $\mu$ such that

$$
\mu^{2}+\lambda=0
$$

That is,

$$
\mu= \pm \sqrt{\lambda} i
$$

(I) (i): For instance, taking $\mu=-\sqrt{\lambda} i$, we have

$$
\begin{gathered}
\psi=e^{-\sqrt{\lambda} i x} \phi, \\
\phi_{2} x+\phi_{1}[-\sqrt{\lambda} i 2 x+2 p+1] \\
+\phi[-i(2 p+1) \sqrt{\lambda}]=f x^{(1 / 2)-p} e^{-\mu x}
\end{gathered}
$$

from (29) and (32).

Applying the operator $N^{v}$ to both members of (36), we find the following equality:

$$
\begin{aligned}
& {\left[\phi_{2} x\right]_{v}+\left\{\phi_{1}[-\sqrt{\lambda} i 2 x+2 p+1]\right\}_{\nu}} \\
& \quad+\{\phi[-i(2 p+1) \sqrt{\lambda}]\}_{\nu}=\left[f x^{(1 / 2)-p} e^{-\mu x}\right]_{\nu}
\end{aligned}
$$


Using (3)-(12), we have

$$
\begin{gathered}
{\left[\phi_{2} x\right]_{v}=x \phi_{2+v}+v \phi_{1+v},} \\
\left\{\phi_{1}[-\sqrt{\lambda} i 2 x+2 p+1]\right\}_{v} \\
=\phi_{1+v}[-\sqrt{\lambda} i 2 x+2 p+1]-\sqrt{\lambda} i 2 v \phi_{v} .
\end{gathered}
$$

Making use of the relations (38), rewrite (37) in the following form:

$$
\begin{aligned}
& \phi_{2+\nu} x+\phi_{1+\nu}[-\sqrt{\lambda} i 2 x+2 p+1+\nu] \\
& -\phi_{\nu}[\sqrt{\lambda} i 2 v+i(2 p+1) \sqrt{\lambda}]=\left[f x^{(1 / 2)-p} e^{i \sqrt{\lambda} x}\right]_{\nu}
\end{aligned}
$$

Choose $v$ such that

$$
v=-p-\frac{1}{2} .
$$

We then have

$$
\begin{gathered}
\phi_{-p+3 / 2} x+\phi_{-p+1 / 2}\left[p+\frac{1}{2}-2 \sqrt{\lambda} i x\right] \\
=\left[f x^{(1 / 2)-p} e^{i \sqrt{\lambda} x}\right]_{-p-1 / 2}
\end{gathered}
$$

from (39).

Next, writing

$$
\phi_{-p+1 / 2}=\omega=\omega(x),
$$

we obtain the following equality from (41):

$$
\begin{aligned}
\omega_{1} & +\omega\left[x^{-1}\left(p+\frac{1}{2}\right)-2 \sqrt{\lambda} i\right] \\
& =\left[f x^{(1 / 2)-p} e^{i \sqrt{\lambda} x}\right]_{-p-1 / 2} x^{-1} .
\end{aligned}
$$

This is an ordinary differential equation of the first order which has a particular solution,

$$
\begin{gathered}
\omega=\left[\left[f x^{(1 / 2)-p} e^{i \sqrt{\lambda} x}\right]_{-p-1 / 2} x^{-1} e^{-2 \sqrt{\lambda} i x} x^{p+1 / 2}\right]_{-1} \\
\quad \times e^{2 \sqrt{\lambda} i x} x^{-p-1 / 2}
\end{gathered}
$$

Making use of the reverse process to obtain $y^{l}$, we finally obtain the solution (17) from (44), (42), (35), and (27).

Inversely, (44) satisfies (43); then

$$
\phi=\omega_{p-1 / 2}
$$

satisfies (41). Therefore, (17) satisfies (16) because we have (27), (35), (44), and (45).

(I) (ii): In the case when $\mu=\sqrt{\lambda} i$, we have

$$
\begin{gathered}
\psi=e^{\sqrt{\lambda} i x} \phi \\
\phi_{2} x+\phi_{1}[2 \sqrt{\lambda} i x+2 p+1] \\
+\phi[(2 p+1) \sqrt{\lambda} i]=f x^{(1 / 2)-p} e^{-i \sqrt{\lambda} x}
\end{gathered}
$$

from (29) and (32).
Applying the operator $N^{v}$ to both members of (47), we have

$$
\begin{aligned}
& \phi_{2+\nu} x+\phi_{1+\nu}[2 \sqrt{\lambda} i x+2 p+1+\nu] \\
& \quad+\phi_{\nu}[\sqrt{\lambda} i(2 p+1) i]=\left(f x^{(1 / 2)-p} e^{-i \sqrt{\lambda} x}\right)_{\nu}
\end{aligned}
$$

Choosing $v$ such that

$$
v=-p-\frac{1}{2}
$$

and replacing

$$
\phi_{-p+1 / 2}=\vartheta=\vartheta(x),
$$

we then obtain

$$
\begin{aligned}
\vartheta_{1} & +\vartheta\left[2 \sqrt{\lambda} i+\left(p+\frac{1}{2}\right) x^{-1}\right] \\
& =\left(f x^{(1 / 2)-p} e^{-i \sqrt{\lambda} x}\right)_{-p-1 / 2} x^{-1}
\end{aligned}
$$

from (48). A particular solution of (51) is given by

$$
\begin{aligned}
\vartheta & =\left[\left(f x^{1 / 2-p} e^{-i \sqrt{\lambda} x}\right)_{-p-1 / 2} x^{-1} e^{2 \sqrt{\lambda} i x} x^{p+1 / 2} d x\right]_{-1} \\
& \times e^{-2 \sqrt{\lambda} i x} x^{-p-1 / 2} .
\end{aligned}
$$

Thus, we have (18) from (52), (50), (46), and (27).

(II) Let $\eta=-p+(1 / 2)$.

With the help of the similar method in (I), replacing $p$ by $-p$ in (I) (i) and (I) (ii), we have other solutions (19) and (20) different from (17) and (18), respectively, if $p \neq 0$.

\section{The Operator $N^{v}$-Method to a \\ Homogeneous Bessel Equation}

Theorem 7. If $y \in \stackrel{o}{\wp}$, just as in Theorem 5 , then the homogeneous Bessel equation

$$
\begin{array}{r}
L[y, x, p]=y_{2}+y\left[\lambda-\frac{p^{2}-1 / 4}{x^{2}}\right]=0, \\
(0<x \leq 1),
\end{array}
$$

has solutions of the forms

$$
\begin{aligned}
& y_{i}=k x^{p+1 / 2} e^{-i \sqrt{\lambda} x}\left\{e^{2 i \sqrt{\lambda} x} x^{-p-1 / 2}\right\}_{p-1 / 2} \\
& y_{u l}=k x^{p+1 / 2} e^{i \sqrt{\lambda} x}\left\{e^{-2 i \sqrt{\lambda} x} x^{-p-1 / 2}\right\}_{p-1 / 2} \\
& y_{v \imath \imath}=k x^{-p+1 / 2} e^{-i \sqrt{\lambda} x}\left\{e^{2 i \sqrt{\lambda} x} x^{p-1 / 2}\right\}_{-p-1 / 2} \\
& y_{i v}=k x^{-p+1 / 2} e^{i \sqrt{\lambda} x}\left\{e^{-2 i \sqrt{\lambda} x} x^{p-1 / 2}\right\}_{-p-1 / 2}
\end{aligned}
$$

for $p \neq 0$, where $k$ is an arbitrary constant. 
Remark 8. In the case when $p=0,(56)$ and (57) coincide with (54) and (55).

Proof. When $f=0$ in Section 2, we have

$$
\begin{aligned}
& \omega_{1}+\omega\left[\left(p+\frac{1}{2}\right) x^{-1}-2 \sqrt{\lambda} i\right]=0, \\
& \vartheta_{1}+\vartheta\left[\left(p+\frac{1}{2}\right) x^{-1}+2 \sqrt{\lambda} i\right]=0,
\end{aligned}
$$

for $\mu=-i \sqrt{\lambda}$ and $\mu=i \sqrt{\lambda}$, instead of (43) and (51).

Therefore, we get (54) for (58) and (55) for (59).

And, for $\eta=-p+(1 / 2)$, replacing $p$ by $-p$ in (58) and (59), we have (56) and (57).

Theorem 9. Let $y \in \stackrel{o}{\wp}$ and $f \in \stackrel{o}{\wp}$ just as in Theorem 5. Then the nonhomogeneous modified Sturm-Liouville equation (16) is satisfied by the fractional differintegrated functions

$$
y=y^{2}+y_{i}
$$

Proof. It is clear by Theorems 5 and 7.

Application 1. If we substitute $p=0, \lambda=1 / 4$, and $f=$ $i x^{-1 / 2} e^{-(i / 2) x}$ in (16), then we obtain the following equation:

$$
y_{2}+y\left(\frac{1}{4}+\frac{1}{4 x^{2}}\right)=i x^{-1 / 2} e^{-(i / 2) x}
$$

and its solution is

$$
\begin{aligned}
y=x^{1 / 2} e^{(-i / 2) x}\{[ & \left(i x^{-1 / 2} e^{-(i / 2) x} x^{1 / 2} e^{(i / 2) x}\right)_{-1 / 2} \\
& \left.\left.\times e^{-i x} x^{-1 / 2}\right]_{-1} e^{i x} x^{-1 / 2}\right\}_{-1 / 2}
\end{aligned}
$$

By performing the necessary operations in (62), we get

$$
y=x^{1 / 2} e^{(-i / 2) x}\left\{\left[\frac{2 i \sqrt{x}}{\sqrt{\pi}} e^{-i x} x^{-1 / 2}\right]_{-1} e^{i x} x^{-1 / 2}\right\}_{-1 / 2},
$$

where Riemann Liouville operator is

$$
\begin{aligned}
{[i]_{-1 / 2} } & =\frac{1}{\Gamma(1 / 2)} \int_{0}^{x} \frac{i}{\sqrt{x-t}} d t=\frac{2 i \sqrt{x}}{\sqrt{\pi}}, \\
y & =x^{1 / 2} e^{(-i / 2) x}\left(-\frac{2 x^{-1 / 2}}{\sqrt{\pi}}\right)_{-1 / 2},
\end{aligned}
$$

and using the definitions of Riemann Liouville operator again, we obtain the following solution:

$$
\begin{gathered}
\left(-\frac{2 x^{-1 / 2}}{\sqrt{\pi}}\right)_{-1 / 2}=-\frac{1}{\Gamma(1 / 2)} \int_{0}^{x} \frac{2 t^{-1 / 2}}{\sqrt{\pi} \sqrt{x-t}} d t=-2, \\
y=-2 x^{1 / 2} e^{-(i / 2) x} .
\end{gathered}
$$
(61):

Now, let us show that the last equality is the solution of

$$
y_{2}=e^{-(i / 2) x}\left[\frac{1}{2} x^{1 / 2}+\frac{1}{2} x^{-3 / 2}+i x^{-1 / 2}\right] .
$$

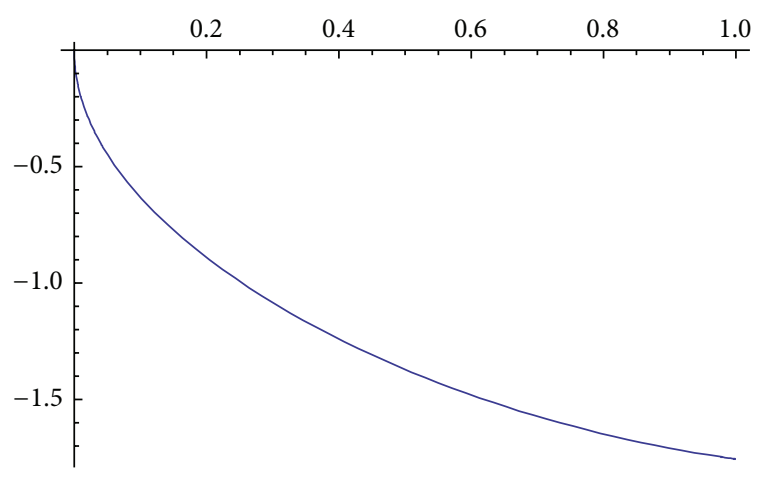

FIGURE 1: Rey.

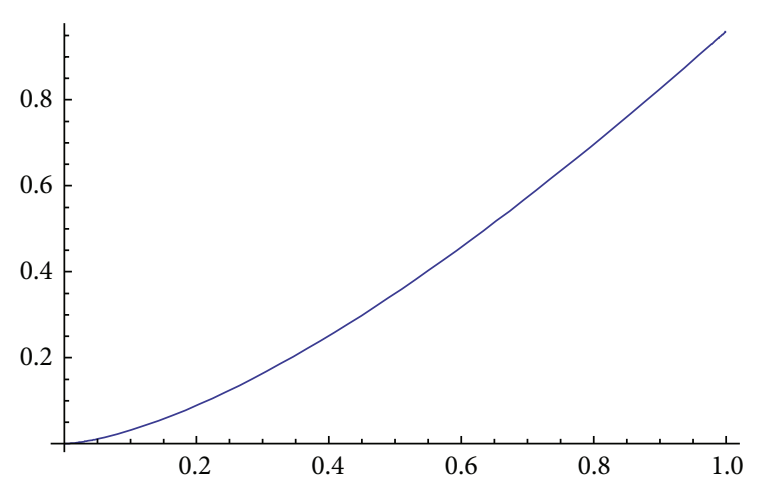

Figure 2: Imy.

Obviously, if (66) and (67) are put in (61), it is satisfied. The graph of the solution of (61) is given in Figures 1 and 2.

Application 2. If we substitute $p=-1$ and $\lambda=0$ in (53), then we obtain the following equation:

$$
y_{2}-\frac{3}{4 x^{2}} y=0 \quad(0<x \leq 1),
$$

and its solution is

$$
y=k x^{-1 / 2}\left\{x^{1 / 2}\right\}_{-3 / 2} .
$$

We prove that $y_{\imath}$ is the solution of (67). With the help of Riemann Liouville operator,

$$
\begin{gathered}
{\left[x^{-1 / 2}\right]_{-1 / 2}=\frac{1}{\Gamma(3 / 2)} \int_{0}^{x} \frac{t^{1 / 2}}{\sqrt{x-t}} d t=\frac{\sqrt{\pi} x^{2}}{4},} \\
y=\frac{k x^{3 / 2} \sqrt{\pi}}{4} .
\end{gathered}
$$

Now, let us show that the last equality is the solution of (67),

$$
y_{2}=\frac{3 k x^{-1 / 2} \sqrt{\pi}}{16} .
$$

Obviously, if (71) and (72) are put into (68), it is satisfied. The graph of the solution of (68) is given in Figure 3. 


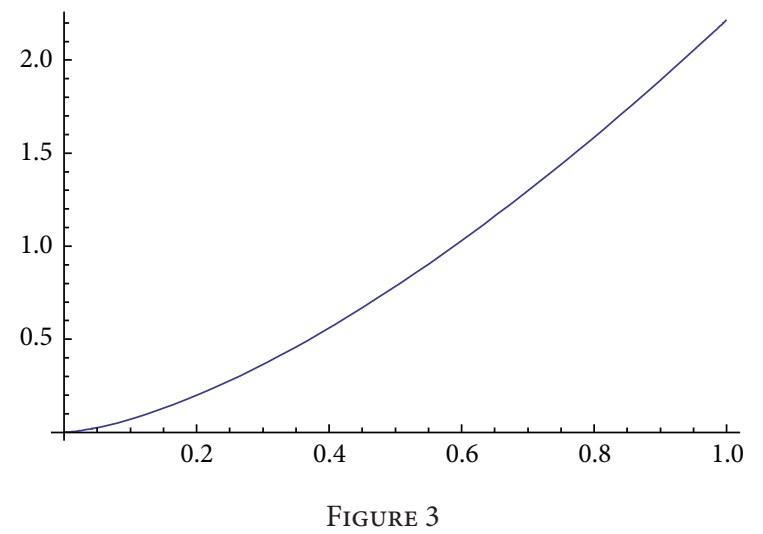

\section{Two Further Cases of Modified Bessel Equation}

Theorem 10. In the similar way as in the previous sections, we can solve the following nonhomogeneous modified Bessel equation:

$$
\begin{aligned}
& y_{2}+y\left[\lambda+\frac{(1 / 4)+p^{2}}{x^{2}}\right]=f, \\
& y_{2}+y\left[-\lambda+\frac{(1 / 4)+p^{2}}{x^{2}}\right]=f,
\end{aligned}
$$

which are obtained by replacing $p$ by ip $(-\lambda$ instead of $\lambda)$ in (16); that is,

$$
\begin{aligned}
& y_{2}+y\left[\lambda+\frac{(1 / 4)-(i p)^{2}}{x^{2}}\right]=f \\
& y_{2}+y\left[-\lambda+\frac{(1 / 4)-(i p)^{2}}{x^{2}}\right]=f .
\end{aligned}
$$

(i) Therefore, the solutions for (74) are given by replacing $p$ by ip in (17), (18), (19), and (20) as follows:

$$
\begin{aligned}
y^{(\imath)}= & x^{p i+1 / 2} e^{-i \sqrt{\lambda} x} \\
\times & \left\{\left[\left(f x^{1 / 2-p i} e^{i \sqrt{\lambda} x}\right)_{-p i-1 / 2} e^{-2 i \sqrt{\lambda} x} x^{p i-1 / 2}\right]_{-1}\right. \\
& \left.\times e^{2 i \sqrt{\lambda} x} x^{-p i-1 / 2}\right\}_{p i-1 / 2}, \\
y^{(2 i)}= & x^{p i+1 / 2} e^{i \sqrt{\lambda} x} \\
\times & \left\{\left[\left(f x^{1 / 2-p i} e^{-i \sqrt{\lambda} x}\right)_{-p i-1 / 2} e^{2 i \sqrt{\lambda} x} x^{p i-1 / 2}\right]_{-1}\right. \\
& \left.\times e^{-2 i \sqrt{\lambda} x} x^{-p i-1 / 2}\right\}_{p i-1 / 2},
\end{aligned}
$$

$$
\begin{aligned}
y^{(\imath i l)}= & x^{-p i+1 / 2} e^{-i \sqrt{\lambda} x} \\
& \times\left\{\left[\left(f x^{1 / 2+p i} e^{i \sqrt{\lambda} x}\right)_{p i-1 / 2} e^{-2 i \sqrt{\lambda} x} x^{-p i-1 / 2}\right]_{-1}\right. \\
& \left.\times e^{2 i \sqrt{\lambda} x} x^{p i-1 / 2}\right\}_{-p i-1 / 2}, \\
y^{(\imath v)}= & x^{-p i+1 / 2} e^{i \sqrt{\lambda} x} \\
& \times\left\{\left[\left(f x^{1 / 2+p i} e^{-i \sqrt{\lambda} x}\right)_{p i-1 / 2} e^{2 i \sqrt{\lambda} x} x^{-p i-1 / 2}\right]_{-1}\right. \\
& \left.\times e^{-2 i \sqrt{\lambda} x} x^{p i-1 / 2}\right\}_{-p i-1 / 2} \cdot
\end{aligned}
$$

(ii) In the same way, for the solutions for (75), substituting the relations (21), and (22) into (75), we have

$$
\phi_{2} x+\phi_{1} 2 v+\phi\left[\left(v^{2}-v+\frac{1}{4}-(p i)^{2}\right) x^{-1}-\lambda x\right]=f x^{1-\nu}
$$

Choose $v$ as follows:

$$
v^{2}-v+\frac{1}{4}+p^{2}=0
$$

That is

$$
v=\frac{1}{2} \pm p i
$$

Let $v=p i+(1 / 2)$. From (21) and (77), we have

$$
\begin{gathered}
y=x^{p i+(1 / 2)} \phi, \\
\phi_{2} x+\phi_{1}(2 p i+1)-\phi \lambda x=f x^{(1 / 2)-p i} .
\end{gathered}
$$

Next, set (29); then (81) is rewritten in the form

$$
\left(e^{\mu x} \psi\right)_{2} x+\left(e^{\mu x} \psi\right)_{1}(2 i p+1)-e^{\mu x} \psi \lambda x=f x^{(1 / 2)-p i} .
$$

Substituting the relations (29) and (31) into (82), we have

$$
\begin{aligned}
& \psi_{2} x+\psi_{1}(2 \mu x+2 p i+1) \\
& \quad+\psi\left[\left(\mu^{2}-\lambda\right) x+(2 p i+1) \mu\right]=f x^{(1 / 2)-i p} e^{-\mu x} .
\end{aligned}
$$

Choose $\mu$ as follows:

$$
\mu^{2}-\lambda=0 .
$$

That is,

$$
\mu= \pm \sqrt{\lambda}
$$

(ii. 1$)$ In the case when $\mu=-\sqrt{\lambda}$, we have

$$
\begin{gathered}
\phi=e^{-\sqrt{\lambda} x} \psi \\
\psi_{2} x+\psi_{1}(-2 \sqrt{\lambda} x+2 p i+1) \\
-\psi[\sqrt{\lambda}(2 p i+1)]=f x^{(1 / 2)-p i} e^{\sqrt{\lambda} x}
\end{gathered}
$$

from (29) and (83). 
Applying the operator $N^{v}$ to both members of (87), we then obtain

$$
\begin{aligned}
& \left(\psi_{2} x\right)_{\nu}+\left[\psi_{1}(-2 \sqrt{\lambda} x+2 p i+1)\right]_{v} \\
& \quad+\{\psi[-\sqrt{\lambda}(2 p i+1)]\}_{v}=\left(f x^{(1 / 2)-p i} e^{\sqrt{\lambda} x}\right)_{\nu}
\end{aligned}
$$

Using (4), (10), and (11), we have

$$
\begin{aligned}
& \psi_{2+v} x+\psi_{1+v}(-2 \sqrt{\lambda} x+2 p i+1+v) \\
& \quad+\psi_{\nu}[-\sqrt{\lambda}(2 p i+1+2 v)]=\left(f x^{(1 / 2)-p i} e^{\sqrt{\lambda} x}\right)_{\nu}
\end{aligned}
$$

Choose $v$ such that

$$
v=-p i-\frac{1}{2}
$$

We then have

$$
\begin{gathered}
\psi_{2-p i-1 / 2} x+\psi_{1-p i-1 / 2}\left[-2 \sqrt{\lambda} x+p i+\frac{1}{2}\right] \\
=\left(f x^{(1 / 2)-p i} e^{\sqrt{\lambda} x}\right)_{-p i-1 / 2}
\end{gathered}
$$

from (89).

Next, writing

$$
\psi_{1 / 2-p i}=u=u(x)
$$

we obtain

$u_{1}+u\left[-2 \sqrt{\lambda}+\left(p i+\frac{1}{2}\right) x^{-1}\right]=\left(f x^{(1 / 2)-p i} e^{\sqrt{\lambda} x}\right)_{-p i-1 / 2} x^{-1}$

from (91). This is an ordinary differential equation of the first order which has a particular solution

$$
\begin{aligned}
u= & {\left[\left(f x^{(1 / 2)-p i} e^{\sqrt{\lambda} x}\right)_{-p i-1 / 2} e^{-2 \sqrt{\lambda} x} x^{p i-1 / 2}\right]_{-1} } \\
& \times e^{2 \sqrt{\lambda} x} x^{-p i-1 / 2} .
\end{aligned}
$$

We finally obtain the solution

$$
\begin{aligned}
y^{(i)}= & x^{p i+1 / 2} e^{-\sqrt{\lambda} x} \\
\times\{[ & \left.\left(f x^{1 / 2-p i} e^{\sqrt{\lambda} x}\right)_{-p i-1 / 2} e^{-2 \sqrt{\lambda} x} x^{p i-1 / 2}\right]_{-1} \\
& \left.\times e^{2 \sqrt{\lambda} x} x^{-p i-1 / 2}\right\}_{p i-1 / 2}
\end{aligned}
$$

from (94), (92), (86) and (80).

(ii. 2) Similarly, in the case when $\mu=\sqrt{\lambda}$, we obtain

$$
\begin{aligned}
y^{(i l)}= & r^{p i+1 / 2} e^{\sqrt{\lambda} x} \\
& \times\left\{\left[\left(f x^{1 / 2-p i} e^{-\sqrt{\lambda} x}\right)_{-p i-1 / 2} e^{2 \sqrt{\lambda} x} x^{p i-1 / 2}\right]_{-1}\right. \\
& \left.\times e^{-2 \sqrt{\lambda} x} x^{-p i-1 / 2}\right\}_{p i-1 / 2}
\end{aligned}
$$

Let $v=-i p+(1 / 2)$. In the same way as in the procedure in (ii), replacing ip by -ip (ii. 1) and (ii. 2), we can obtain $y^{(i n)}$ and $y^{(\imath v)}$.

Theorem 11. In the homogeneous case for (74) with $f=0$, using the solutions (54), (55), (56), and (57) and replacing $p$ by pi, we obtain

$$
\begin{gathered}
y_{(\imath)}=\alpha x^{p i+1 / 2} e^{-i \sqrt{\lambda} x}\left(e^{2 i \sqrt{\lambda} x} x^{-p i-1 / 2}\right)_{p i-1 / 2}, \\
y_{(\imath \imath)}=\alpha x^{p i+1 / 2} e^{i \sqrt{\lambda} x}\left(e^{-2 i \sqrt{\lambda} x} x^{-p i-1 / 2}\right)_{p i-1 / 2}, \\
y_{(i \imath l)}=\alpha x^{-p i+1 / 2} e^{-i \sqrt{\lambda} x}\left(e^{2 i \sqrt{\lambda} x} x^{p i-1 / 2}\right)_{-p i-1 / 2} \\
y_{(i v)}=\alpha x^{-p i+1 / 2} e^{i \sqrt{\lambda} x}\left(e^{-2 i \sqrt{\lambda} x} x^{p i-1 / 2}\right)_{-p i-1 / 2}
\end{gathered}
$$

for $p \neq 0$, where $\alpha$ is an arbitrary constant.

\section{Conclusion}

The $N$-fractional calculus operator $N^{v}$-method is applied to the nonhomogeneous and homogeneous Bessel equation. Explicit fractional solutions of Bessel equations are obtained. Furthermore, similar solutions were obtained for the modified same equation by using the method.

\section{Acknowledgment}

The authors sincerely thank the reviewers for their valuable suggestions and useful comments.

\section{References}

[1] L. Adam, Fractional Calculus: History, Definitions and Applications For the Engineer, 2004.

[2] A. A. Kilbas, H. M. Srivastava, and J. J. Trujillo, Theory and Applications of Fractional Differential Equations, vol. 204 of North-Holland Mathematical Studies, Elsevier (North-Holland) Science Publishers, Amsterdam, The Netherlands, 2006.

[3] I. Podlubny, Fractional Differential Equations: An Introduction To Fractional Derivatives, Fractional Differential Equations, Methods of Their Solution and Some of Their Applications, Mathematics in Science and Enginering, vol. 198, Academic Press, New York, NY, USA, 1999.

[4] S. G. Samko, A. A. Kilbas, and O. I. Marichev, Fractional Integrals and Derivatives: Theory and Applications Translated From the Russian: Integrals and Derivatives of Fractional Order and Some of Their Applications, "Nauka i Tekhnika", Minsk, (1987), Gordon and Breach Science Publishers, Reading, UK, 1993.

[5] K. B. Oldham and J. Spanier, The Fractional Calculus, Akademic Press, New York, NY, USA, 1974.

[6] K. Nishimoto, Fractional Calculus, vol. I, Descartes Press, Koriyama, Japan, 1984.

[7] K. Nishimoto, Fractional Calculus, vol. II, Descartes Press, Koriyama, Japan, 1987.

[8] K. Nishimoto, Fractional Calculus, vol. III, Descartes Press, Koriyama, Japan, 1989. 
[9] K. Nishimoto, Fractional Calculus, vol. IV, Descartes Press, Koriyama, Japan, 1991.

[10] K. Nishimoto, Fractional Calculus, vol. V, Descartes Press, Koriyama, Japan, 1996.

[11] K. Nishimoto and S. S. de Romero, " $N$-fractional calculus operator $N^{v}$ method to nonhomogeneous and homogeneous Whittaker Equations," Journal of Fractional Calculus, vol. 9, pp. 17-22, 1996.

[12] K. Nishimoto, An Essence of Nishimoto's Fractional Calculus (Calculus of the 21st Century): Integrations and Differentiations of Arbitrary Order, Descartes Press, Koriyama, Japan, 1991.

[13] M. Coz and P. Rochus, "Translation kernels for velocity dependent interactions," Journal of Mathematical Physics, vol. 18, no. 11, pp. 2232-2240, 1976.

[14] A. Erdely, W. Magnus, F. Oberhettinger, and F. G. Tricomi, Tables of Integral Transform, vol. II, McGraw-Hill, New York, NY, USA, 1954.

[15] A. Erdely, "Axially symmetric potentials and fractional integration," Journal of the Society for Industrial and Applied Mathematics, vol. 13, pp. 216-228, 1965.

[16] A. Weinstein, "Generalized axially symmetric potential theory," Bulletin of the American Mathematical Society, vol. 59, pp. 2038, 1953.

[17] B. Ross, Fractional Calculus and Its Applications, Conference Proceedings held at the University of New Haven, June 1974, Springer, New York, NY, USA, 1975.

[18] S. Salinas De Romero and H. M. Srivastava, "An application of the $N$-fractional calculus operator method to a modified Whittaker equation," Applied Mathematics and Computation, vol. 115, no. 1, pp. 11-21, 2000.

[19] H. M. Srivastava and R. G. Buschman, Theory and Applications of Convolution Integral Equations, vol. 79 of Kluwer Series on Mathematics and Its Applications, Kluwer Academic Publishers, Dordrecht, The Netherlands, 1992.

[20] H. M. Srivastava, S. Owa, and K. Nishimoto, "Some fractional differintegral equations," Journal of Mathematical Analysis and Applications, vol. 106, no. 2, pp. 360-366, 1985.

[21] H. Koyunbakan and E. S. Panakhov, "Transformation operator for singular Sturm-Liouville equations," International Journal of Applied Mathematics, vol. 14, pp. 135-143, 2003. 


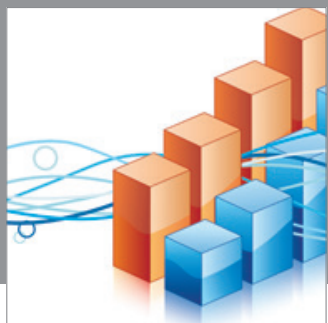

Advances in

Operations Research

mansans

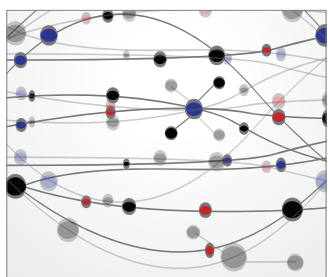

The Scientific World Journal
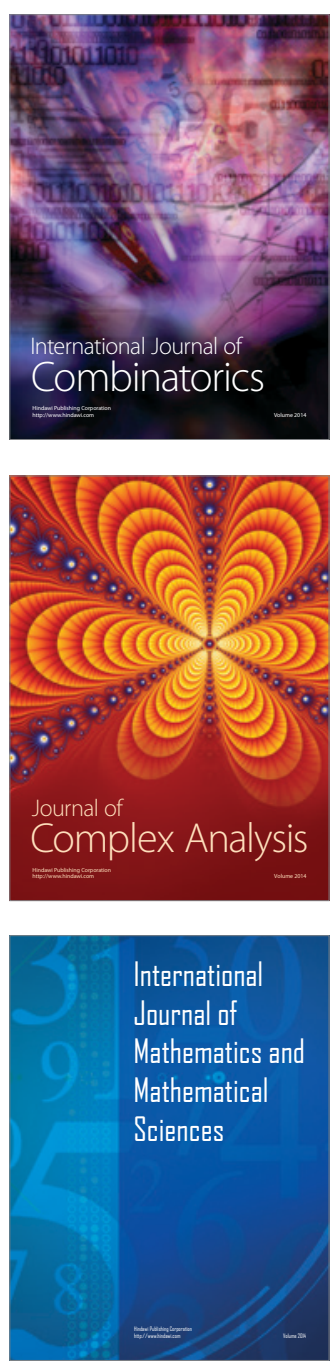
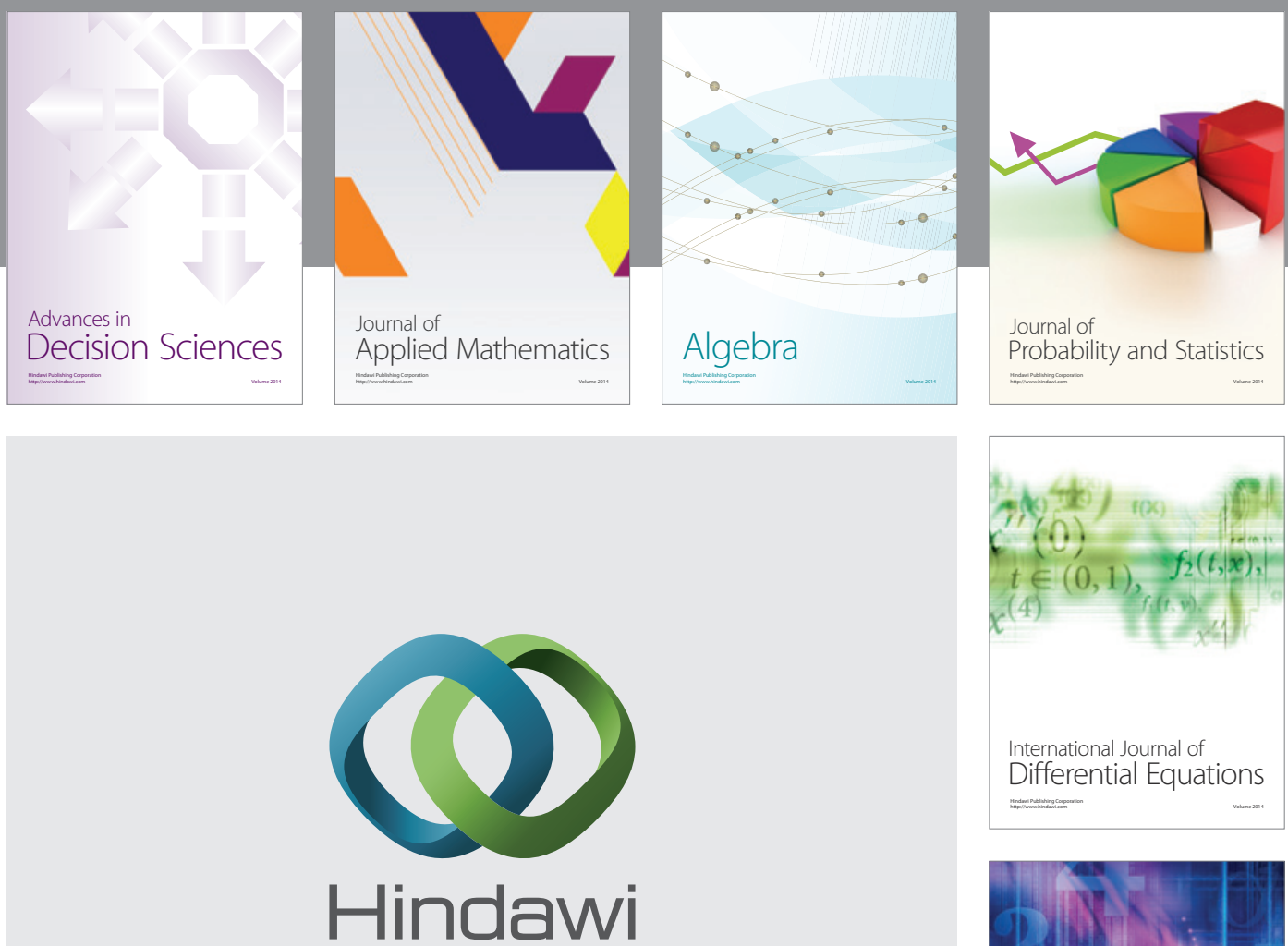

Submit your manuscripts at http://www.hindawi.com
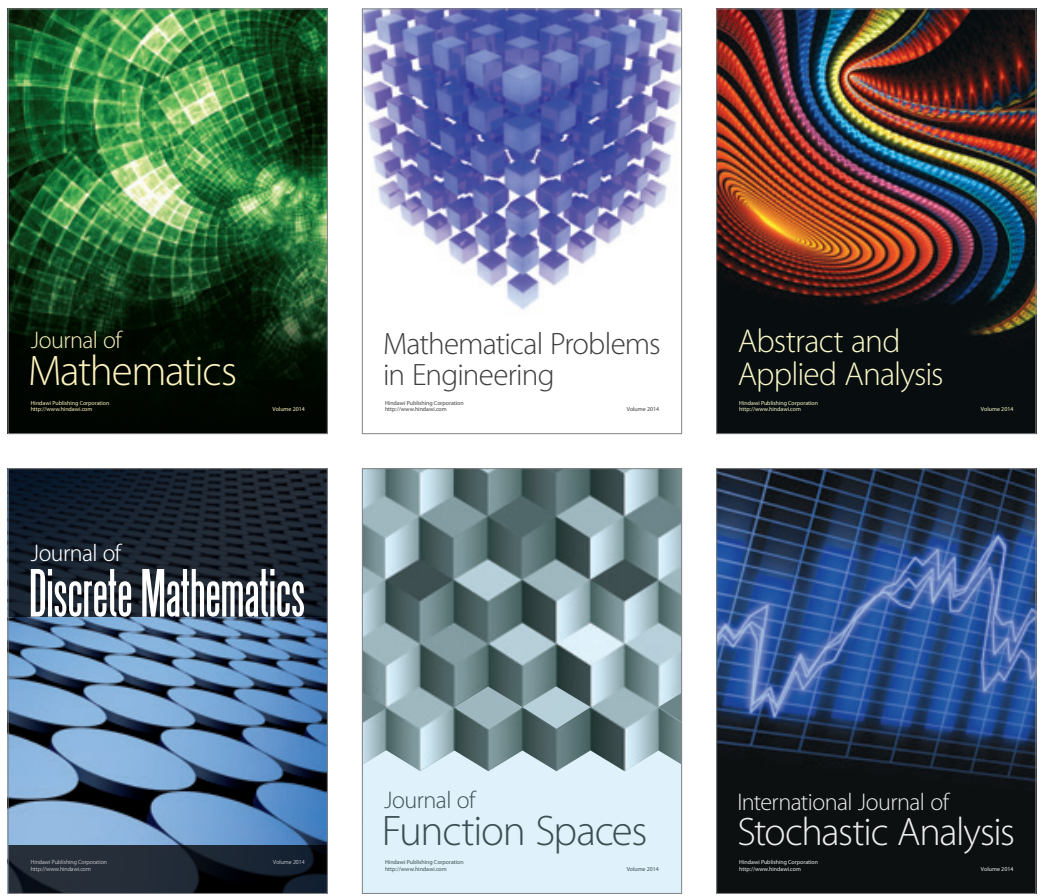

Journal of

Function Spaces

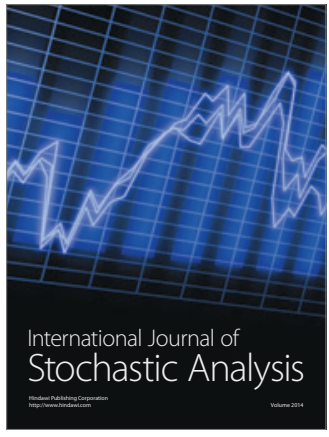

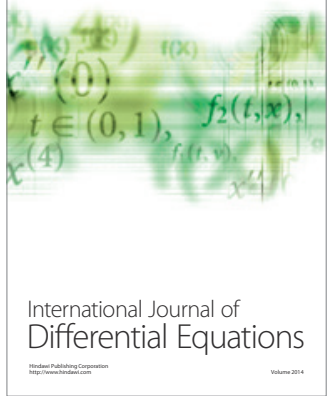
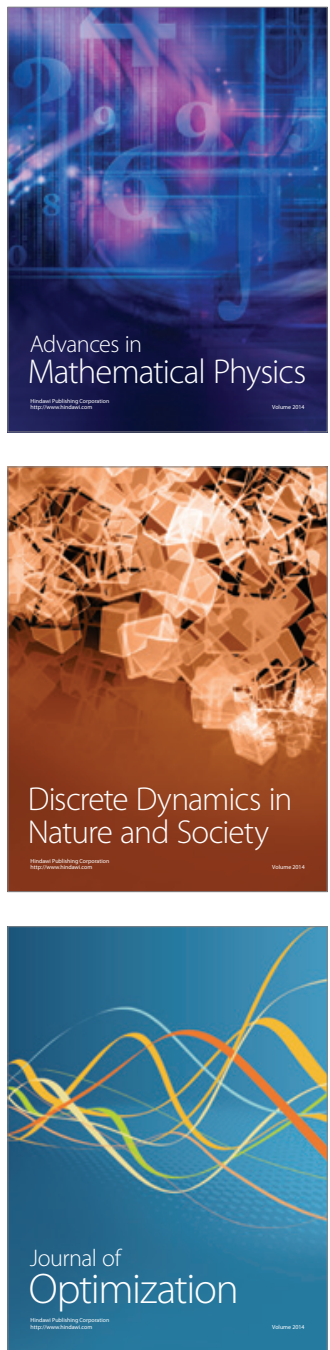